\title{
Four Wave Mixing Effects in Gain-Equalized Distributed Fiber Raman Amplifiers
}

\author{
Marcelo A. Soto and Ricardo Olivares
}

Department of Electronics, Universidad Técnica Federico Santa María, Casilla 110-V, Valparaíso, Chile

\begin{abstract}
The degradation of the performance of distributed fiber Raman amplifiers (DFRAs) is shown to be dependent on the interaction between pump-pump four-wave mixing (FWM) and stimulated Raman scattering (SRS), by means of numerical simulations. We verify that FWM processes, including depletion and parametric gain, generate new sources of SRS and a redistribution of the pump power along the fiber. Results show impairments on the performance of DFRAs such as loss of flatness on the spectral gain and reduction on the Raman gain and presence of FWM products within the transmission band.
\end{abstract}

Index Terms - Four wave mixing, distributed fiber Raman amplifiers, WDM transmission systems.

\section{INTRODUCTION}

Recent investigations have demonstrated that distributed fiber Raman amplification is the most efficient all-optical amplification method, due to its ultra-wide bandwidth, flexible wavelength operation, low noise and capacity to mitigate fiber nonlinearities. However, the performance of a distributed fiber Raman amplifier (DFRA) is determined both by the model and the method used to design. In transmission system applications, the main target of a DFRA is to compensate the attenuation of the channels occurred when they are propagated along the optical fiber; in this sense, a DFRA must provide flat gain for all the WDM channels. To satisfy this requirement, several design techniques have been proposed in order to optimize the spectral gain profile of the amplifier [1]-[3]. The objective of all these optimization methods is to find the spectral position and the input power of each pump in the amplifier. Some of these methods can be also applied to design cascades of DFRAs, in such a way that each amplifier of the cascade provides a flat output power spectrum for all the involved channels. The optimization problem is divided in two parts which are carried out sequentially by solving the mathematical model presented in [1], which describes the gain and depletion power due to stimulated Raman scattering (SRS). The first part allows one to find the wavelength of each pump wave, and the second one, to determine the input power of each of them. However in practice, if the designed amplifier is implemented in a fiber with low chromatic dispersion, four-wave mixing (FWM) and parametric amplification can be produced. By considering that the used mathematical model does not consider the interaction of FWM and SRS, the output power spectrum will be different with respect to the expected one.
In this work we analyze, by using numerical simulations, the effects of FWM in DFRAs, which are previously designed to obtain a flat gain, and operating with pump waves located near the zero dispersion wavelength $\left(\lambda_{Z D}\right)$. The objective is to analyze how an equalized spectrum can be affected by generated FWM products, parametric gain, and new interactions between FWM products, pumps and channels.

\section{MATHEMATICAL MoDEL}

The numerical model used in this work can be derived from the equations presented in [1]-[4], where the mathematical models for SRS and FWM are obtained. Thus, the propagation equation for the complex envelope of the electric field $\left(A_{F}\right)$ at frequency $f_{F}$, considering SRS and FWM processes, is:

$$
\begin{aligned}
& \frac{d A_{F}(z)}{d z}=-\frac{\alpha_{F}}{2} A_{F}(z) \\
& +\sum_{f_{m}>f_{F}} \frac{g_{R}\left(f_{F}, f_{m}\right)}{2 A_{e f f} K_{e f f}\left(f_{F}, f_{m}\right)}\left|A_{m}(z)\right|^{2} A_{F}(z) \\
& -\sum_{f_{m}<f_{F}} \frac{f_{F}}{f_{m}} \frac{g_{R}\left(f_{F}, f_{m}\right)}{2 A_{e f f} K_{e f f}\left(f_{F}, f_{m}\right)}\left|A_{m}(z)\right|^{2} A_{F}(z) \\
& +j \frac{1}{3} \gamma \sum_{i, j \neq k}\left\{K_{f w m}\left(f_{i}, f_{j}, f_{k}\right) D_{i j k}\right. \\
& \left.\quad \times A_{i}(z) A_{j}(z) A_{k}^{*}(z) \exp \left(-\beta_{i j k} z\right)\right\} \\
& +j \frac{1}{3} \gamma \sum_{p, r \neq q}\left\{K_{f w m}\left(f_{F}, f_{q}, f_{r}\right) D_{p q r}\right. \\
& \left.\quad \times A_{p}(z) A_{q}^{*}(z) A_{r}(z) \exp \left(\Delta \beta_{p q r} z\right)\right\}
\end{aligned}
$$

In (1) the conditions to be satisfied are $f_{F}=f_{i}+f_{j}-f_{k}$ (FWM with parametric gain) and $f_{p}=f_{F}+f_{q}-f_{r}$ (FWM depletion); $g_{R}$ is the Raman gain coefficient, $\gamma$ is the nonlinear coefficient, $D_{i j k}$ is the degeneracy factor, $\Delta \beta_{i j k}$ is the linear phase-mismatch, $K_{\text {eff }}$ and $K_{f w m}$ are the polarization factors for SRS and FWM, respectively. $K_{\text {eff }}$ is 0.5 or 1 , depending if the waves at frequency $f_{F}$ and $f_{m}$ have identical or random polarization states, respectively [1]. $K_{f w m}$ is 1 when the waves involved on FWM process have parallel linear polarizations, and $(1 / 2)^{0.5}$ or $(3 / 8)^{0.5}$ for partially degenerate and nondegenerate FWM with random polarization states, respectively [6] 
One important aspect of equation (1) is that it describes the interaction of FWM and SRS simultaneously and can be solved by a simple numerical method. The main difference of this model with respect to others previously proposed [7]-[8], is that it includes a complete description of both nonlinearities, including the Raman gain/depletion, parametric gain, depletion due to FWM and generation of new FWM waves. It avoids the use of iterative methods required to get convergence in the solution when these nonlinearities are solved separately [7]; therefore, it also allows one to reduce computing time. In addition, with this model is not necessary to use closed mathematical expressions based on undepleted conditions for the waves, which can be inappropriate approximations when a strong SRS-FWM interaction exists.

\section{FWM EFFICIENCY}

In order to understand the presence of FWM in DFRAs we need to analyze the efficiency of each FWM product generated in the amplifier. Equation (2) describes the wellknown FWM efficiency [5]:

$$
\eta=\frac{\alpha^{2}}{\alpha^{2}+\Delta \beta^{2}}\left[1+\frac{4 e^{-\alpha L} \sin ^{2}(\Delta \beta L / 2)}{\{1-\exp (-\alpha L)\}^{2}}\right]
$$

Where $\alpha$ is the attenuation coefficient; $L$ is the fiber length; and $\Delta \beta$ is the linear phase-mismatch factor, which can be obtained as follows:

$$
\begin{aligned}
\Delta \beta & =\frac{2 \pi \lambda_{0}^{2}}{c}\left\{\left(\begin{array}{ll}
f_{i} & f_{k}
\end{array}\right)\left(f_{j} \quad f_{k}\right)\right\} D_{c}\left(f_{o}\right) \\
& -\frac{\pi \lambda_{0}^{4}}{c^{2}}\left\{\left(f_{i}-f_{k}\right)\left(\begin{array}{ll}
f_{j} & f_{k}
\end{array}\right)\right\}\left\{\begin{array}{lll}
f_{i} & f_{j} & 2 f_{o}
\end{array}\right\} S_{c}\left(f_{o}\right)
\end{aligned}
$$

Where $f_{i}, f_{j}$ and $f_{k}$ are the frequencies involved in the FWM process, $c$ is the light velocity and, $D_{c}$ and $S_{0}$ are the chromatic dispersion and dispersion slope of the fiber at a reference frequency $f_{0}\left(\lambda_{0}\right)$, respectively. Equation (3) can be reduced for spectral region with low chromatic dispersion as follows:

$-\Delta \beta=\frac{\pi \lambda_{0}^{4}}{c^{2}}\left\{\left(\begin{array}{ll}f_{i} & f_{k}\end{array}\right)\left(\begin{array}{ll}f_{j} & f_{k}\end{array}\right)\right\}\left\{\begin{array}{lll}f_{i} & f_{j} & 2 f_{o}\end{array}\right\} S_{c}\left(f_{o}\right)$

In this case, the reference frequency corresponds to the frequency $f_{0}\left(f_{0}=c / \lambda_{Z D}\right)$. By using the definition of equivalent frequency separation [5], we have:

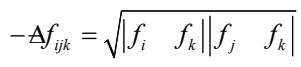

We can also define the spectral separation of $f_{0}$ with respect to the middle frequency between $f_{i}$ and $f_{j}$ :

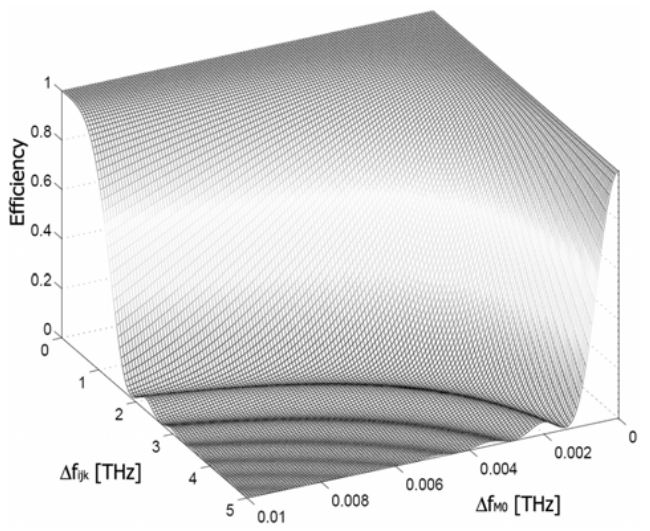

Fig 1. FWM efficiency as function of $\Delta f_{i j k}$ and $\Delta f_{M 0}$.

$$
\Delta f_{M 0}=\frac{f_{i}+f_{j}}{2}-f_{0}
$$

Therefore, combining (5) and (6) into (4) we obtain:

$$
\Delta \beta=\frac{2 \pi \lambda_{0}^{4}}{c^{2}} f_{i j k} \quad f_{M 0} S_{c}\left(f_{o}\right)
$$

Fig. 1 depicts the FWM efficiency described by equation (2) as a function of $\Delta f_{i j k}$ and $\Delta f_{M 0}$. If we take into account that $f_{i}, f_{j}$ and $f_{k}$ are three Raman pumps of a DFRA, there are two ways to get a strong pump-pump FWM interaction $(\eta=1)$. The first is when $\Delta f_{i j k}=0$, however this condition occurs when $f_{i}=f_{k}$ or $f_{j}=f_{k}$, which are the specific cases of SPM or XPM. The second way occurs when the condition $\Delta f_{M 0}=0$ is satisfied. For degenerated FWM, it occurs when the degenerated pump coincides with the $\lambda_{Z D}\left(f_{i}=f_{j}=c / \lambda_{Z D}\right)$. For non-degenerated FWM, it occurs when $c / \lambda_{Z D}$ coincides with the frequency in the middle of $f_{i}$ and $f_{j}\left(c / \lambda_{Z D}=\left(f_{i}+f_{j}\right) / 2\right)$.

It is important to remark that strong FWM products can be obtained even if the efficiency is not equal to 1. A high enough efficiency could also produce a strong FWM product due to the high pump power available (the power of the FWM product depends of the multiplication of the efficiency and the involved pump powers).

From both conditions presented above, note that the condition $\Delta f_{i j k} \approx 0$ is difficult to satisfy because usually the Raman pumps are spectrally distributed in a broad band. However, $\Delta f_{M O} \approx 0$ easily could occur depending on the number of Raman pumps and the $\lambda_{Z D}$ (it is independent of the spectral spacing of the pumps). Taking into account that conditions, and analyzing Fig. 1, we can also deduce that different spectral regions will exist within the pump band, where if $\lambda_{Z D}$ takes place, at least one high efficient FWM product will be generated. 


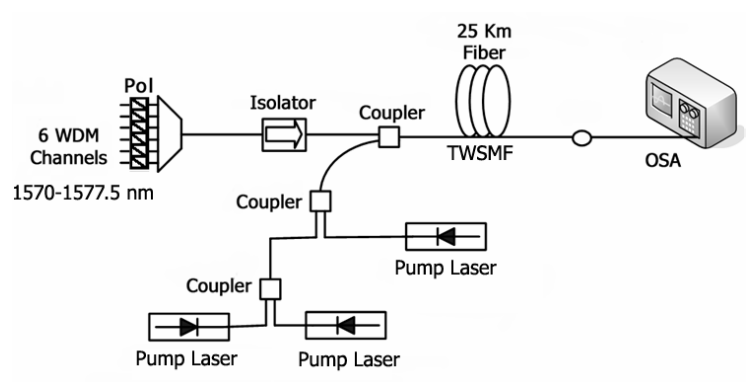

Fig 2. Experimental setup.

\section{EXPERIMENTAL RESULTS}

In order to show the strong presence of pump-pump FWM in a DFRA, we performed the experimental setup shown in Fig. 2. The DFRA amplifies six lineally polarized WDM channels, in the range 1570-1577.5 nm with input power of 1 $\mu \mathrm{W} / \mathrm{ch}$. It uses three depolarized pump lasers at 1450, 1480 and $1488 \mathrm{~nm}$, with 100,180 and $150 \mathrm{~mW}$ input power, respectively (these values correspond to the powers launched into the fiber, i.e. coupler and connector losses are undertaken). The fiber is a TrueWave (TWSMF) with $25-\mathrm{km}$ length and $\lambda_{Z D}=1498 \mathrm{~nm}$.

Once the fiber is characterized in terms of Raman gain and loss, its parameters can be plugged into equation (1), which is then solved for the conditions previously described.

The simulated results have good agreement with the experimental measurements as shown in Fig. 3. The differences $(<2 \mathrm{~dB}$ on average) can be attributed mainly by possible longitudinal random fluctuations of some parameters along the fiber, such as effective area and chromatic dispersion (note the high sensitivity of the FWM process with respect to $A_{e f f}$ and $\lambda_{Z D}$ for low dispersion regions).

\section{FWM EFFECTS IN GAIN-EQUALIZED DFRAS}

To evaluate the effects of FWM in the spectral profile of gain-equalized DFRA, three different WDM transmission systems are previously designed. Each system is composed of one forward pumped DFRA, which is designed through an optimization method based on a genetic algorithm [3]. The purpose of this design method is to find the wavelength and power of each pump in order to obtain the flattest net gain in each WDM system. The mathematical model used in the design is commonly used for this target, which just describes SRS and spontaneous Raman scattering, neglecting FWM.

The first transmission system, over $50 \mathrm{~km}$ of TWSMF, is composed of 20 depolarized WDM channels in the range $1540-1560 \mathrm{~nm}$, and with an input power of $0.5 \mathrm{~mW} / \mathrm{ch}$. As result of the design, three Raman pumps were obtained as shown Table I, obtaining an optimized spectrum with $0.21 \mathrm{~dB}$ of ripple. However, the low chromatic dispersion of the TWSMF at around the pump wavelengths will produce strong

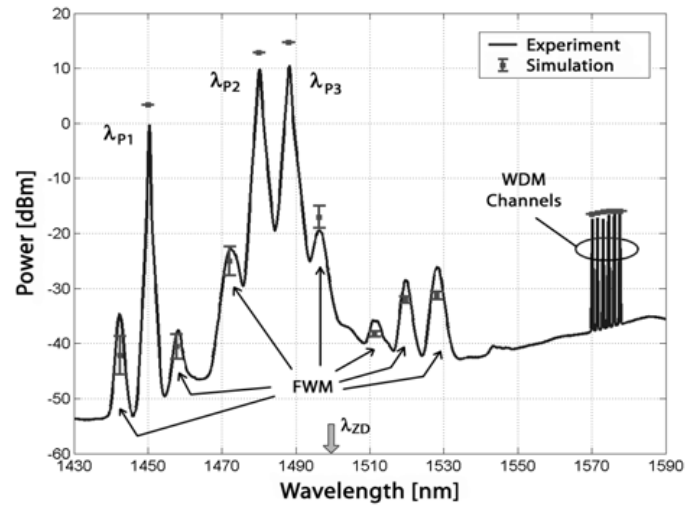

Fig 3. Theoretical and experimental results.

TABLE I

OPTIMIZED PUMPS FOR DFRA WITH 20NM-BANDWIDTH

\begin{tabular}{|l|l|l|l|}
\hline$\lambda[\mathrm{nm}]$ & 1420.28 & 1438.14 & 1462.52 \\
\hline Power $[\mathrm{mW}]$ & 160.93 & 92.24 & 99.61 \\
\hline
\end{tabular}

pump-pump FWM interactions. Therefore, several FWM products will be generated from the three pumps.

From equations (2), (6) and (7), note that an interesting situation occurs when the $\lambda_{Z D}$ is located in the middle of the pumps frequency range, i.e., if $\lambda_{Z D}=1441.58 \mathrm{~nm}$. Fig. 4 shows the output power spectrum of the transmission system. We can note the high output power of the non-degenerated FWM produced at $1445.03 \mathrm{~nm}$. Due to its high power and spectral position, it acts as a pump for the amplifier, affecting the designed power equalization. Fig. 5 depicts a comparison between the equalized output power spectrum and the obtained spectrum including pump-pump FWM and FWM-toSRS interactions. It can be note that more than $7.0 \mathrm{~dB}$ of ripple is obtained when FWM is undertaken.

From equation (6), it is also possible to see that highly efficient FWM products can be produced when the $\lambda_{Z D}$ coincides with a pump wavelength (degenerated FWM processes). For instance, when the $\lambda_{Z D}=1463.52 \mathrm{~nm}$ the FWM product with the longest wavelength has a unitary efficiency. This FWM product, in addition to its high efficiency, is strongly amplified by SRS due to its spectral position. Therefore, an additional depletion of the pumps is produced due to the Raman amplification of the two FWM products with the longest wavelength, as shown Fig. 6. The depletion of the pumps is transferred to the gain of the DFRA, as shown Fig. 7. On average, it corresponds to a penalty of $\sim$ $3 \mathrm{~dB}$ in the gain of the amplifier.

In order to analyze broader bandwidth systems, the second transmission system is composed of $20 \mathrm{WDM}$ channels in the range 1540-1580 $\mathrm{nm}$. By using the previously mentioned genetic algorithm, a ripple of $0.36 \mathrm{~dB}$ in the output power was obtained with the four pumps shown in Table II.

Because the pumps of the amplifier are spectrally spread, the FWM products are also generated in a wide bandwidth. In particular, when $\lambda_{Z D}=1476.77 \mathrm{~nm}$, strong FWM products will 


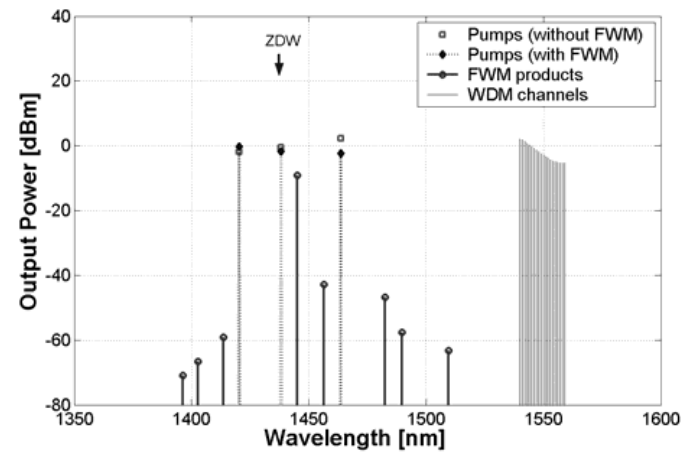

Fig 4. Output power spectrum of the DFRA with $20 \mathrm{~nm}$ bandwidth, when $\lambda_{Z D}=1441.58 \mathrm{~nm}$ ).

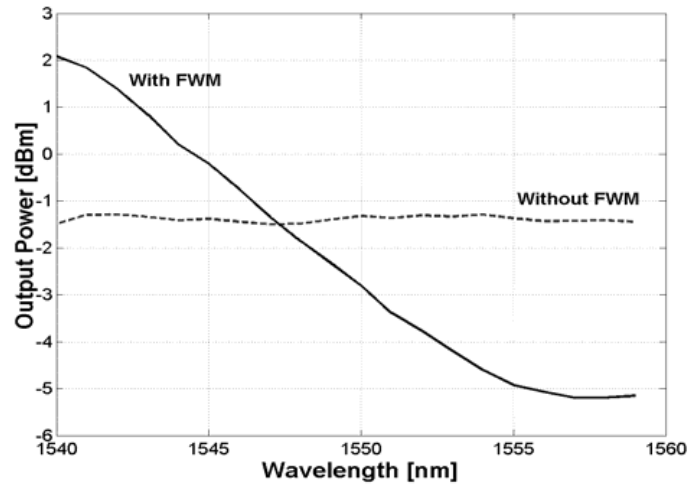

Fig 5. Output power spectrum of WDM channels. DFRA with $20 \mathrm{~nm}$-bandwidth and $\lambda_{Z D}=1441.58 \mathrm{~nm}$.

TABLE II

OPTIMIZED PUMPS FOR DFRA WITH 40NM-BANDWIDTH

\begin{tabular}{|l|l|c|l|l|}
\hline$\lambda[\mathrm{nm}]$ & 1405.32 & 1428.31 & 1445.00 & 1476.77 \\
\hline Power[mW] & 175.80 & 108.18 & 47.74 & 34.50 \\
\hline
\end{tabular}

be generated in the longer wavelengths. Fig. 8 depicts this situation, where one strong FWM wave is produced within the WDM band. Fig. 9 shows the strong wave in the middle of the WDM channels, and the decreased net gain of the system due to the involved transfer of energy. Penalties of $3.7 \mathrm{~dB}$ in the net gain and a ripple of $\sim 2 \mathrm{~dB}$ are obtained.

Finally, a system with 40 channels is designed in the range 1520-1600 nm. Due to the wide bandwidth of the system, six pumps are obtained by the genetic algorithm (see Table III) to produce a ripple of $0.84 \mathrm{~dB}$ at the output. This also produces a broad spectral region where the FWM products will appear. When the $\lambda_{Z D}$ is placed near the pumps with longer wavelengths, a strong Raman amplification will be achieved by the most efficient FWM products. As shown Fig. 10, several strong FWM products will be generated within the WDM band. Because the pump at $1490.05 \mathrm{~nm}$ gives its energy to the most efficient FWM products, it experiences a strong depletion (due to FWM). This produces a detrimental effect in the gain of the channels with longer wavelength, as

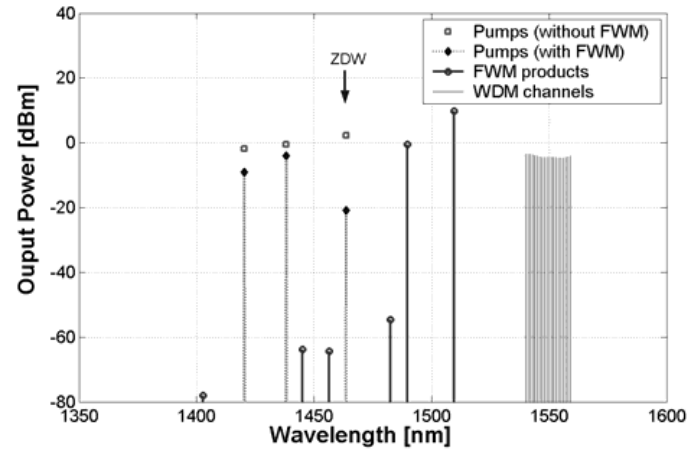

Fig 6. Output power spectrum of the DFRA with 20nmbandwidth, when $\lambda_{Z D}=1463.52 \mathrm{~nm}$ ).

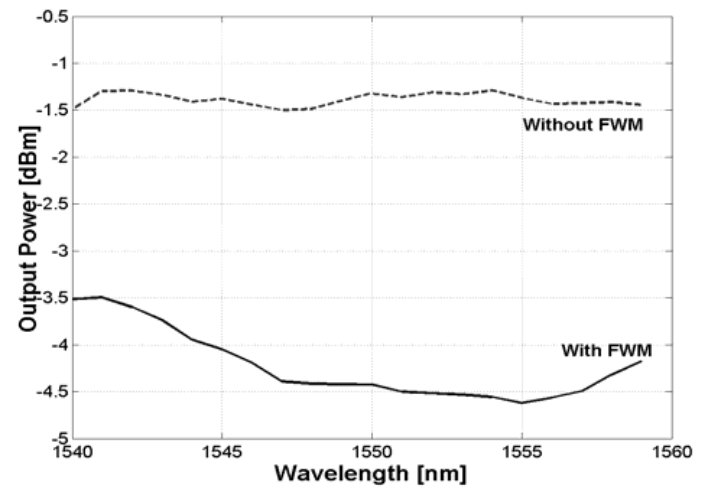

Fig 7. Output power spectrum of WDM channels. DFRA with $20 \mathrm{~nm}$-bandwidth and $\lambda_{Z D}=1463.52 \mathrm{~nm}$.

TABLE III

OPTIMIZED PUMPS FOR DFRA WITH 80NM-BANDWIDTH

\begin{tabular}{|l|l|l|l|}
\hline$\lambda[\mathrm{nm}]$ & 1404.80 & 1420.10 & 1431.72 \\
\hline Power [mW] & 53.43 & 144.58 & 113.36 \\
\hline$\lambda[\mathrm{nm}]$ & 1444.41 & 1466.72 & 1490.05 \\
\hline Power $[\mathrm{mW}]$ & 60.93 & 34.38 & 16.77 \\
\hline
\end{tabular}

shown Fig. 11. However, the channels with shorter wavelength are affected just by the small depletion of the pumps with shorter wavelengths (additional depletion produced by the transfer of energy from pump to FWM products by SRS processes).

It is important to remark that in this work we have shown the most relevant results for the three analyzed DFRAs. However, in each transmission system several positions of $\lambda_{Z D}$ also produce similar results.

\section{CONCLUSIONS}

In this work, the interaction between SRS and FWM in DFRAs was studied by using a comprehensive mathematical model. It was verified that FWM processes (including new FWM waves, depletion and parametric gain) generate new sources of SRS and a redistribution of the pump power along 


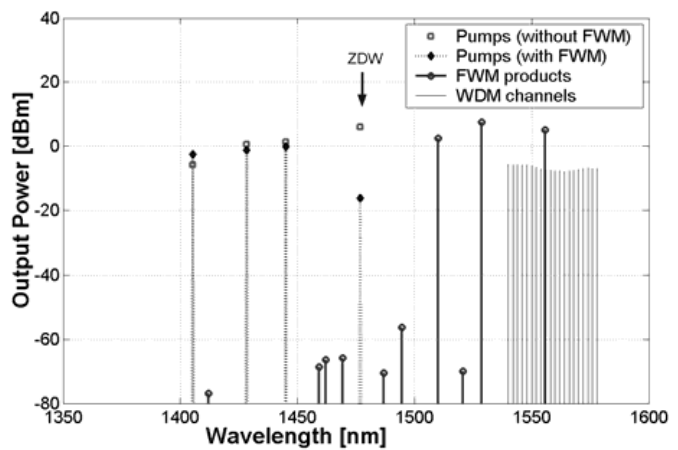

Fig 8. Output power spectrum of the DFRA with 40nmbandwidth, when $\lambda_{Z D}=1476.77 \mathrm{~nm}$ ).

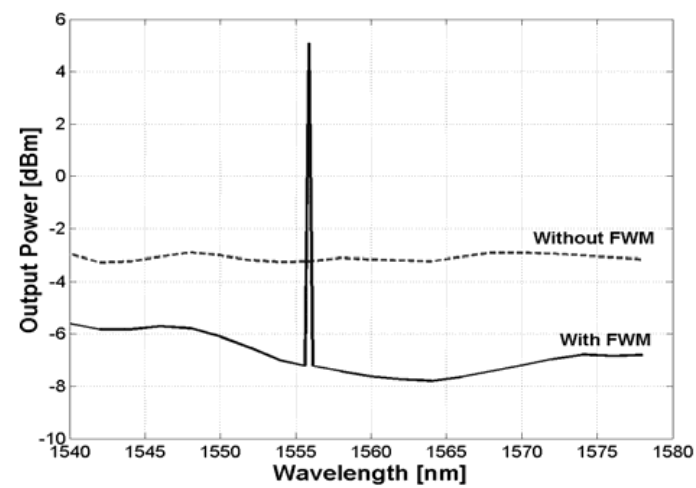

Fig 9. Output power spectrum of WDM channels. DFRA with $40 \mathrm{~nm}$-bandwidth and $\lambda_{Z D}=1476.77 \mathrm{~nm}$.

the fiber. Performance degradation in distributed amplified systems can be produced due to these interactions; this degradation can be manifested as a combination of some effects, such as loss of gain-equalization, penalties in the net gain of the amplifier (due to pump depletions produced by new SRS-FWM interactions) and the presence of strong FWM products within the transmission band. It is concluded that FWM processes must be considered in the design of DFRAs in order to assure an expected performance.

\section{ACKNOWLEDGEMENT}

Authors thanks to the Chilean Agency Conicyt (Fondicyt project \#1010437) and to Universidad Técnica Federico Santa María (DGIP project \#230729) for the support in this work.

\section{REFERENCES}

[1] V. E. Perlin and H. G. Winful, "Optimal Design of Flet-Gain Wide-Band fiber Raman Amplifier," J. Lightw. Technol., vol. 20, no. 2, pp. 250-254, February 2002.

[2] J. Chen, X. Liu, C. Lu, Y. Wang and Z. Li, "Design of Multistage Gain-Flattened Fiber Raman Amplifiers," J. Lightw. Technol., vol. 24, no. 2, pp. 935-944, February 2006.

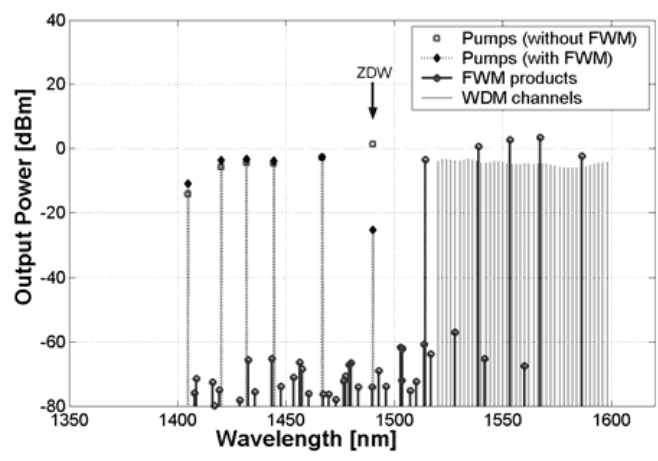

Fig 10. Output power spectrum of the DFRA with 80nmbandwidth, when $\lambda_{Z D}=1490.05 \mathrm{~nm}$ ).

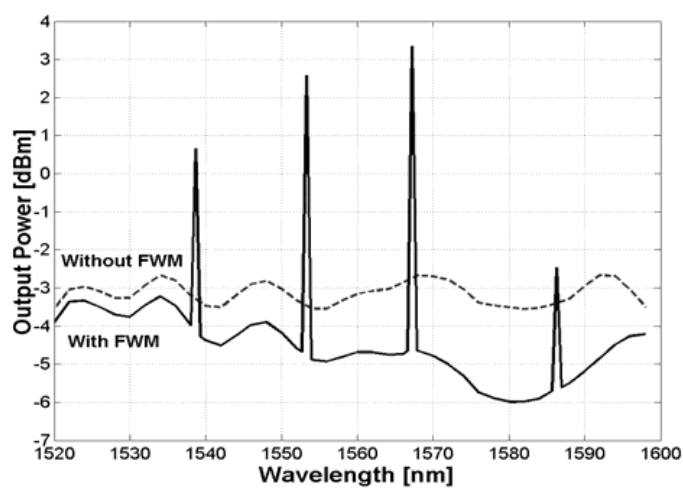

Fig 11. Output power spectrum of WDM channels. DFRA with $80 \mathrm{~nm}$-bandwidth and $\lambda_{Z D}=1490.05 \mathrm{~nm}$.

[3] H. Soto, V. Pincheira and R. Olivares, "Optimal Design Based on Genetic Algorithm of Distributed Fiber Raman Amplifier Cascades", 5th Iberoamerican Meeting on Optics and 8th Latin American Meeting on Optics, Lasers, and Their Applications, Proceedings of SPIE, vol. 5622, pp. 364-367, SPIE, Bellingham, WA, 2004.

[4] K. O. Hill, D. C. Johnson, B. Kawasaki and R. I. MacDonald, "Cw Three-Wave Mixing in Single-Mode Optical fibers," $J$. Appl. Phys., vol. 49, no.10, pp. 5098-5106, October 1978.

[5] N. Shibata, R. Braun and R. Waarts, "Phase-Mismatch Dependence of Efficiency of Wave Generation Through FourWave Mixing in a Single-Mode Optical Fiber," IEEE J. Quantum Electron., vol. QE-23, no. 7, pp. 1205-1210, July 1987.

[6] K. Inoue, "Polarization Effect on Four-Wave Mixing Efficiency in a Single-Mode Fiber," IEEE J. of Quantum Electron., vol. 28, no. 4, pp. 883-894, April 1992.

[7] J.-C. Bouteiller, L. Leng and C. Headley, "Pump-Pump FourWave Mixing in Distributed Raman Amplified Systems," J. Lightw. Technol., vol. 22, no. 3, pp. 723-732, March 2004.

[8] F. Vanholsbeeck, S. Coen, P. Emplit, M. Haelterman and T. sylvestre, "Coupled-Mode Analysis of Stimulated Raman Scattering and Four-Wave Mixing in Wavelength-Division Multiplexed Systems," Optics Communications, vol. 250, pp. 191-201, 2005. 